\title{
Universiteit
}

Leiden

The Netherlands

\section{Vibrationally induced two-level systems in single-molecule junctions}

Thijssen, W.H.A.; Djukic, D.; Otte, A.F.; Bremmer, R.H.; Ruitenbeek, J.M. van

\section{Citation}

Thijssen, W. H. A., Djukic, D., Otte, A. F., Bremmer, R. H., \& Ruitenbeek, J. M. van. (2006). Vibrationally induced two-level systems in single-molecule junctions. Physical Review Letters, 97(22), 226806. doi:10.1103/PhysRevLett.97.226806

Version: $\quad$ Not Applicable (or Unknown)

License: $\quad$ Leiden University Non-exclusive license

Downloaded from: https://hdl.handle.net/1887/62365

Note: To cite this publication please use the final published version (if applicable). 


\title{
Vibrationally Induced Two-Level Systems in Single-Molecule Junctions
}

\author{
W. H. A. Thijssen, D. Djukic, A. F. Otte, R. H. Bremmer, and J. M. van Ruitenbeek \\ Kamerlingh Onnes Laboratory, Leiden University, P.O. Box 9504, 2300 RA Leiden, The Netherlands
}

(Received 18 July 2006; published 1 December 2006)

\begin{abstract}
Single-molecule junctions are found to show anomalous spikes in $d I / d V$ spectra. The position in energy of the spikes is related to local vibration mode energies. A model of vibrationally induced twolevel systems reproduces the data very well. This mechanism is expected to be quite general for singlemolecule junctions. It acts as an intrinsic amplification mechanism for local vibration mode features and may be exploited as a new spectroscopic tool.
\end{abstract}

DOI: 10.1103/PhysRevLett.97.226806

A single atom or molecule with an almost transparent single conductance channel leading to a conductance near the conductance quantum $2 e^{2} / h\left(=1 G_{0}\right)$ can be contacted to leads. Conduction electrons can pass through such junction ballistically for low bias voltages since the mean free path of the electrons is much larger than the size of the contact. However, the contact is not entirely ballistic in the sense that once the excess energy of the conduction electrons becomes equal to or larger than the energy of a local mode of the contact, the electrons can scatter inelastically by exciting a local mode. This results in the case of a perfectly transmitting single channel contact to a small decrease in the conductance, since the forward traveling electrons are backscattered due to the energy loss in the inelastic scattering process. Differential conductance $(d I / d V)$ measurements have identified vibration modes of single molecules in an atomic contact [1]. This technique, also called point contact spectroscopy is analogous to inelastic electron tunneling spectroscopy (IETS) for single molecules $[2,3]$, with the difference that the conductance in the latter case increases due to the opening of an additional conductance channel.

In this Letter we present the observation of anomalous spikes, rather than steps, in $d I / d V$ measurements on various single-molecule contacts. We present a model that involves two-level systems, which describes our data very well. It may be used as a new spectroscopic tool for identifying molecular vibration modes in single-molecule junctions.

We create atomic contacts using a mechanically controlled break junction setup in cryogenic vacuum at $4.2 \mathrm{~K}$ (see Ref. [4] for a detailed description). Break junctions for the metals $\mathrm{Au}, \mathrm{Ag}, \mathrm{Pt}$, and $\mathrm{Ni}$ have been investigated with the molecules $\mathrm{H}_{2}, \mathrm{D}_{2}, \mathrm{O}_{2}, \mathrm{C}_{2} \mathrm{H}_{2}, \mathrm{CO}, \mathrm{H}_{2} \mathrm{O}$, and benzene. In most of these cases regular vibration mode spectra displaying a step down in conductance have been observed, but for all systems anomalous spectral features as displayed in Fig. 1 were also found.

In order to admit these molecules to the metal atomic contacts at $4.2 \mathrm{~K}$, the insert is equipped with a capillary that has a heating wire running all along its interior to prevent premature condensation of the gasses. The amount of gas
PACS numbers: 73.63.Rt, 63.22.+m, 81.07.Nb, 85.65.+h

admitted is of order $10 \mu \mathrm{mol}$. Previous measurements have clearly demonstrated that it is possible to capture a single molecule in the atomic junction and measure its vibration modes $[1,5]$.

Figure 1(a) shows a $d I / d V$ spectrum of a single $\mathrm{CO}$ molecule contacted by Pt leads. The observation of spikes that dominate the spectrum is surprising. Pt-CO-Pt contacts at a conductance of $G \simeq 1 G_{0}$, as for Pt-H-H-Pt contacts [1], typically display steplike decreases, at the same energies as the spikes, in $d I / d V$ when conduction electrons are backscattered due to energy loss in exciting a local vibration mode [6]. Figure 2(c) shows an example of a regular $d I / d V$ spectrum for a Pt-D-D-Pt contact. The $I-V$ curve of Fig. 1(b) shows clear fluctuations in the voltage window in which the peaks in $d I / d V$ appear. At high voltages the fluctuations have disappeared and the junction displays a slightly higher conductance.

We have evidence that the anomalies are related to vibration modes of the single molecule. First, for the Pt- $\mathrm{H}_{2}$ system the spikes in $d I / d V$ typically appear at the same energies as the transverse vibration mode energies of a hydrogen molecule [5]. Figures 2(a)-2(c) show a sequence of spectra taken when a Pt-D-D-Pt contact was slightly stretched over $0.2 \AA$. The small bumps on the vibration mode shoulders in Fig. 2(a) at $\pm 48 \mathrm{mV}$ evolve into full peaks in Fig. 2(b) and subsequently into a regular vibration mode spectrum for a deuterium molecule in between Pt contacts in Fig. 2(c). Additionally we have
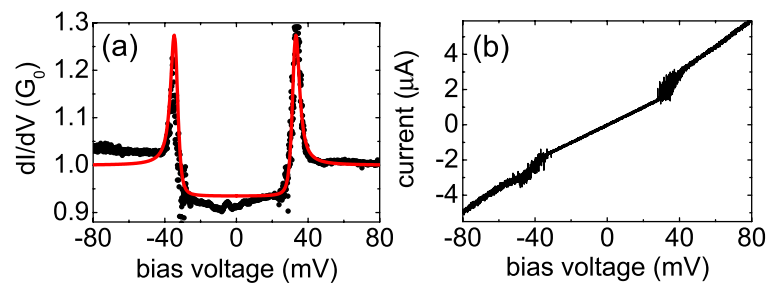

FIG. 1 (color online). (a) $d I / d V$ spectrum on a CO molecule bridging a Pt contact displaying symmetric positive spikes. The red (gray) curve shows a fit by the model discussed in the text [21]; (b) $I-V$ measurement on a $\mathrm{CO}$ molecule bridging a $\mathrm{Pt}$ contact. 


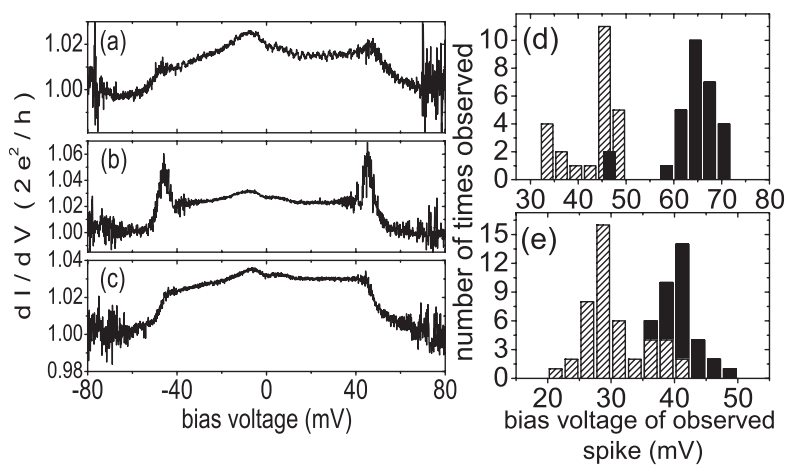

FIG. 2. Panels (a)-(c) display the gradual change of $d I / d V$ spectra of a Pt-D-D-Pt contact; panels (b) and (c) show spectra taken for the same junction as (a) after stretching by 0.1 and $0.2 \AA$, respectively. Panels (d) and (e) show histograms of peak positions in spectra of junctions for $\mathrm{Pt}_{-} \mathrm{H}_{2}(\mathrm{~d}), \mathrm{Au}-\mathrm{H}_{2}$ (e) (solid bars) and $\mathrm{Pt}_{-} \mathrm{D}_{2}(\mathrm{~d}), \mathrm{Au}-\mathrm{D}_{2}$ (e) (hatched bars). All data were obtained at $T=4.2 \mathrm{~K}$.

introduced hydrogen to atomic gold contacts and subsequently measured $d I / d V$. The spectra show similar anomalous spikes as for $\mathrm{Pt}-\mathrm{H}_{2}$. The positions of the spikes observed in $d I / d V$ spectra for $\mathrm{Pt}$ and Au with $\mathrm{H}_{2}$ and $\mathrm{D}_{2}$ are shown as histograms in Figs. 2(d) and 2(e). It can be clearly seen that the histogram maxima have shifted with the square root of the mass ratio, which is expected for molecular vibration mode energies.

Spikes in $d I / d V$ point to abrupt changes in the conductance of the contacts in small voltage windows. Since similar spikes have been observed for different molecules, this suggests that it involves a general feature of singlemolecule junctions. The "noise" visible in Fig. 1(b) is a result of two-level fluctuations, which are excited at high bias. They are not excited directly in view of the connection with vibration mode energies shown in Fig. 2. Reference [7] has shown that by exciting internal vibrations of a pyrrolidine molecule on a metal surface, the molecule can flip to a different conformation. The different conformations have different conductances leading to a dip in $d I / d V$. This forms a basis for our proposed explanation.

We attribute the abrupt changes in contact conductance to abrupt switching between two slightly different local geometrical configurations that have different conductances. Current induced two-level systems in mesoscopic contacts have been studied in detail in the past (see, e.g., [8-10]). In those cases spikes are observed only in the second derivative $d^{2} I / d V^{2}$, in contrast to spikes in $d I / d V$ in our measurements. Since the anomalous spikes appear around the vibration mode energies, we assume that the switching is induced by intramolecular vibrations. STM studies on single molecules on metal surfaces have clearly shown, e.g., that by exciting an internal vibration mode of a molecule the lateral hopping rate of a $\mathrm{CO}$ molecule [11], the rotation of a $\mathrm{O}_{2}$ molecule [12] or a $\mathrm{C}_{2} \mathrm{H}_{2}$ molecule [13] can be increased by 2 orders of magnitude. In our case the molecules are chemically linked by two leads preventing the molecules of jumping away and the current is much higher, leading to orders of magnitude higher rates.

We derive a simple model of a vibrationally induced two-level system (VITLS) that reproduces the shape of the anomalous spikes in our $d I / d V$ spectra very well. The model is schematically depicted in Fig. 3. The molecule experiences a double well potential landscape with ground states $\Psi_{0}$ and $\Psi_{1}$ in the two energy minima. The two energy minima are separated by a large potential barrier that prevents tunneling between the two ground states. The molecule in the contact can be vibrationally excited by conduction electrons to the state $\Psi^{e}$. In order to displace the molecule, the barrier for the reaction coordinate has to be overcome. The direction of the internal vibration needs to be in the direction of the reaction coordinate (i.e., the movement) of the molecule [14]. In the case of the Pt- $\mathrm{D}_{2}$ junctions the spikes have been observed only at energies corresponding to vibrations perpendicular to the current direction (i.e., transverse modes). The longitudinal vibration mode around $90 \mathrm{meV}$ [5] never showed anomalous spikes. In our quasi-one-dimensional junctions the transverse modes are the ones that have the proper reaction coordinates to induce changes in the geometry of the junctions.

The expression for the current passing through the junction can be approximately written as

$$
I(V)=\left(n_{0} \sigma_{0}+n_{1} \sigma_{1}+n_{e} \frac{\left(\sigma_{0}+\sigma_{1}\right)}{2}\right) V .
$$

We assume that the conductance $\sigma_{0}$ of well 0 and $\sigma_{1}$ of well 1 are different. A step in $I(V)$ and thus a peak in $d I / d V$ can be obtained once $\Psi_{1}$ becomes populated. The total current is determined by the sum of the products of the occupation numbers with their respective conductances. For simplicity, we take the average of $\sigma_{0}$ and $\sigma_{1}$ to be

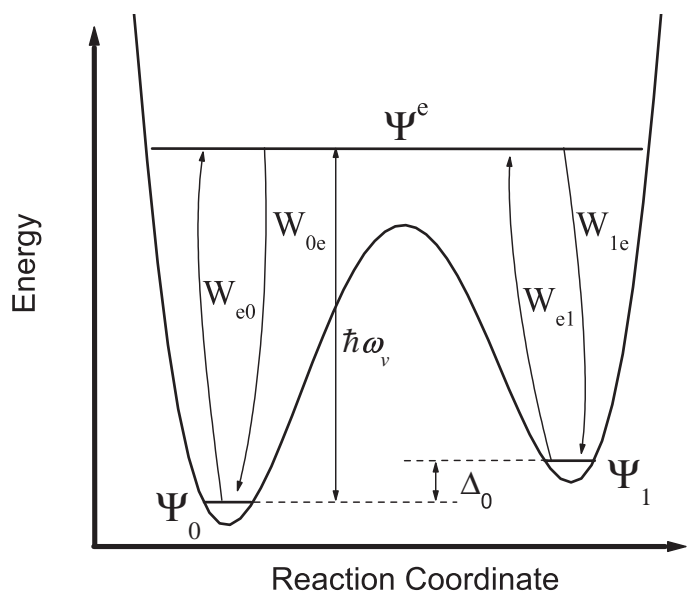

FIG. 3. Energy landscape of a molecule in an atomic contact. The molecule can be vibrationally excited and relax into the other energy minimum. 
the conductance of the excited state. In order to determine the shape of the $d I / d V$ spectrum, the dependence of $n_{0}, n_{1}$, and $n_{e}$ on $V$ has to be determined. Let us therefore consider dynamic equilibrium of the occupation numbers of all levels involved in the system as displayed in Fig. 3 in terms of the transition rates,

$$
\begin{gathered}
\frac{d n_{0}}{d t}=-W_{e 0}+W_{0 e}=0, \quad \frac{d n_{1}}{d t}=-W_{e 1}+W_{1 e}=0 \\
\frac{d n_{e}}{d t}=-W_{0 e}-W_{1 e}+W_{e 0}+W_{e 1}=0 \\
n_{0}+n_{1}+n_{e}=1 .
\end{gathered}
$$

Here $W_{f i}$ represent the transition rates from states $i$ to states $f$. We define the energy difference between the two ground states (Fig. 3) to be $\Delta_{0}$. The vibration mode energy $\hbar \omega_{v}$ is much larger than $\Delta_{0}$ and $k_{B} T \leq \Delta_{0}$ to keep the system well defined in the ground state for low bias voltages. The transition rates $W$ can now be determined. Consider as an example the rate $W_{e 0}$ :

$$
W_{e 0}=n_{0} \int_{-\infty}^{+\infty} \gamma f(E, e V)\left[1-f\left(E-\hbar \omega_{v}, e V\right)\right] d E
$$

We assumed here that the electron density of states is flat over the energy window under consideration. Here $\gamma$ is the electron-phonon coupling and $f(E, e V)$ are the nonequilibrium electron distribution functions. All other rates can be expressed in a similar way. After considering the low temperature limit, $\frac{\hbar \omega_{v}}{k_{B} T}, \frac{\left(\hbar \omega_{v}-\Delta_{0}\right)}{k_{B} T} \gg 1$, and limiting to positive biases only we can solve the integrals of Eq. (3):

$$
\begin{array}{r}
\int_{-\infty}^{+\infty} f(E, e V)[1-f(E+\Delta E, e V)] d E=\Delta E+\frac{1}{4} \frac{\Delta E-e V}{e^{(\Delta E-e V) / k_{B} T}-1} \quad \text { when } \Delta E>0, \\
\frac{1}{4} \frac{-\Delta E-e V}{e^{(-\Delta E-e V) / k_{B} T}-1} \quad \text { when } \Delta E<0
\end{array}
$$

With the results of Eq. (4) we can calculate the rates of Eq. (2). The set of linear equations can be solved analytically, and the resulting occupation numbers can be inserted in the expression for the current (1). Figure 4(a) shows a measurement and fit for a $\mathrm{H}_{2}$ molecule in a Pt contact, while Fig. 4(b) shows the evolution of the occupation numbers, which clearly shows a sharp drop in $n_{0}$ and a rise in $n_{1}$ once the vibration mode energy is reached. Figures 4(c) and 4(d) show spectra and fits for $\mathrm{H}_{2}$ in a

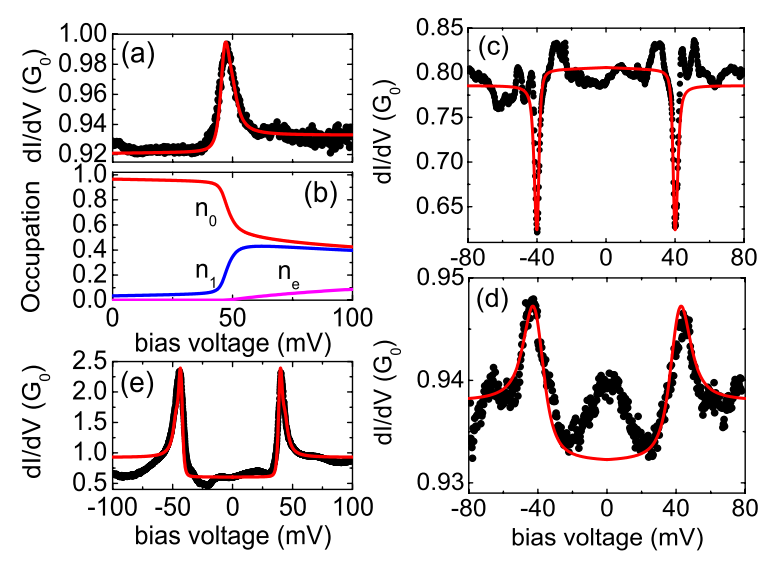

FIG. 4 (color online). Several fits of VITLS for singlemolecule junction contacts and atomic chains: (a) $\mathrm{H}_{2}$ in a $\mathrm{Pt}$ contact; (b) evolution of occupation numbers $n_{0}, n_{1}, n_{e}$ for the fit of (a); (c) $\mathrm{H}_{2}$ in a Au atomic chain; (d) $\mathrm{H}_{2}$ in a Au atomic chain; (e) $\mathrm{O}_{2}$ in a Ni contact. The bath temperature was $5 \mathrm{~K}$ in all cases except in (d), where the contact was heated to $20 \mathrm{~K}$. For fit parameters, see [21].
$\mathrm{Au}$ atomic chain at different temperatures. Hydrogen molecules incorporated in atomic gold chains have recently been observed [15]. In Fig. 4(d) the regular phonon mode of the gold atoms in the chain at $\pm 15 \mathrm{meV}$ can be resolved next to the VITLS spikes. Figure 4(c) shows clear negative spikes even when large conductance fluctuations are present. These conductance fluctuations arise from interference of electron waves that scatter on defects and impurities close to the contacts [16]. Often, the regular vibration mode signal is masked by these fluctuations for conductances $<1 G_{0}$, but the spikes clearly show. Figure 4(e) shows a spectrum and fit for $\mathrm{O}_{2}$ in a Ni contact, and a fit was also added in Fig. 1(a), indicating that the model is not limited to hydrogen. The difference in binding energy between the two ground states $\Psi_{0}$ and $\Psi_{1}$ is $1-3 \mathrm{meV}$, which is reasonable if one assumes two different geometrical configurations of the molecule that display slightly different conductances. $\Delta_{0}$ is clearly larger than the fitted temperatures, which are about $0.3-0.6 \mathrm{meV}(\sim 4-7 \mathrm{~K})$ in all cases except for the spectrum of Fig. 4(d), for which the fitted temperature is $1.6 \mathrm{meV}(\sim 20 \mathrm{~K})$ in agreement with the bath temperature. This implies that for $V \ll V m$ the system is almost entirely in state $\Psi_{0}$ [see Fig. 4(b)], which is a prerequisite for obtaining a sufficiently large decrease of $n_{0}$ and increase of $n_{1}$ when $V$ reaches $V_{m}$. The origin of the somewhat higher fitted temperatures compared to the bath temperature remains unclear, but was also observed in the width of regular vibration mode spectra $[1,5]$.

The model described above involves vibrational excitation above the barrier. We have elaborated a similar 
model in which the excited states are weakly tunneling coupled. The resulting fits are not qualitatively different [17].

Recently, STM measurements on a hydrogen covered $\mathrm{Cu}(111)$ surface yielded very similar anomalous $d I / d V$ spikes [18]. The mechanism is probably related but not identical to ours because the peak positions showed strong dependence on hydrogen coverage of the surface. Moreover, the observed two-level fluctuations were observed to remain in the $\sigma_{1}$ state above the threshold voltage. Possibly the geometry of the experiment allows for the molecules to be excited into positions away from the STM tip, after which they are slow to return.

The IETS measurements by Wang et al. [19] on a selfassembled monolayer of octanedithiols have been discussed [20] in view of the unexpected shape of the features in $d^{2} I / d V^{2}$. Several of the stronger features have the shape of peaks in the first derivative, $d I / d V$, but still at the energies of known vibration mode energies for the molecules. Our model produces a good fit to the data by Wang et al., but the temperature in the fit is much higher than the experimental temperature. This may be attributed to inhomogeneous broadening in the ensemble of molecules measured.

We conclude that the observed spikes in $d I / d V$ measurements on single-molecule junctions are most probably due to vibrationally induced two-level systems. Our simple model fits the data very well. It will be interesting to investigate potential energy landscapes for singlemolecule junctions in atomistic models in order to obtain a more concrete picture of the states involved. The VITLS acts as an intrinsic amplification mechanism providing a strong spectroscopic signature for local vibration modes, and the anomalous spikes in $d I / d V$ are expected to be a general feature of single-molecule junctions. It can therefore be exploited as a new spectroscopic tool in analogy to the "action spectrum" proposed by Komeda et. al. [11]. Specifically, Au atomic chains with $\mathrm{H}_{2}$, which very rarely show regular vibration mode spectra, have a vibration mode around $42 \mathrm{meV}$, as seen from Fig. 2(e).

We would like to thank W. Y. Wang, M. A. Reed, J. A. Gupta, and A. J. Heinrich for valuable discussions and communicating their $d I / d V$ spectra. This work is part of the research program of the "Stichting FOM," partially financed through the SONS programme of the European
Science Foundation, which is also funded by the European Commission, Sixth Framework Programme, and was also supported by the European Commission TMR Network program DIENOW.

[1] R. H. M. Smit et al., Nature (London) 419, 906 (2002).

[2] B. C. Stipe, M. A. Rezaei, and W. Ho, Science 280, 1732 (1998).

[3] H. Park et al., Nature (London) 407, 57 (2000).

[4] N. Agraït, A. Levy Yeyati, and J. M. van Ruitenbeek, Phys. Rep. 377, 81 (2003).

[5] D. Djukic et al., Phys. Rev. B 71, 161402(R) (2005).

[6] D. Djukic and J. M. van Ruitenbeek (to be published).

[7] J. Gaudioso, L. J. Lauhon, and W. Ho, Phys. Rev. Lett. 85, 1918 (2000).

[8] R. J.P. Keijsers, O. I. Shklyarevskii, and H. van Kempen, Phys. Rev. B 51, 5628 (1995).

[9] D. C. Ralph and R. A. Buhrman, Phys. Rev. B 51, 3554 (1995).

[10] A. Halbritter, L. Borda, and A. Zawadowski, Adv. Phys. 53, 939 (2004).

[11] T. Komeda, Y. Kim, M. Kawai, B. N. J. Persson, and H. Ueba, Science 295, 2055 (2002).

[12] B. C. Stipe, M. A. Rezaei, and W. Ho, Science 279, 1907 (1998).

[13] B. C. Stipe, M. A. Rezaei, and W. Ho, Phys. Rev. Lett. 81, 1263 (1998).

[14] B. N. J. Persson and H. Ueba, Surf. Sci. 502-503, 18 (2002).

[15] Sz. Csonka, A. Halbritter, and G. Mihály, Phys. Rev. B 73, 075405 (2006).

[16] B. Ludoph and J. M. van Ruitenbeek, Phys. Rev. B 61, 2273 (2000).

[17] D. Djukic, Ph.D. thesis, Leiden University, 2006.

[18] J. A. Gupta, C. P. Lutz, A. J. Heinrich, and D. M. Eigler, Phys. Rev. B 71, 115416 (2005).

[19] W. Y. Wang, T. Lee, I. Kretzschmar, and M. A. Reed, Nano Lett. 4, 643 (2004).

[20] M. Galperin, M. A. Ratner, and A. Nitzan, J. Chem. Phys. 121, 11965 (2004).

[21] Fit parameters $\left[\sigma_{0}, \sigma_{1}, \hbar \omega_{v}, \Delta_{0}, T\right]$ : for Fig. 1(a), $\left[0.93 G_{0}, 1.07 G_{0}, 33 \mathrm{meV}, 2.5 \mathrm{meV}, 6.5 \mathrm{~K}\right]$; for Fig. 4(a), $\left[0.923 G_{0}, 0.946 G_{0}, 47 \mathrm{meV}, 3.0 \mathrm{meV}, 8 \mathrm{~K}\right]$; for Fig. 4(c), $\left[0.803 G_{0}, 0.765 G_{0}, 40 \mathrm{meV}, 1.0 \mathrm{meV}, T=4 \mathrm{~K}\right]$; for Fig. 4(d), $\left[0.924 G_{0}, 0.954 G_{0}, 42 \mathrm{meV}, 1.5 \mathrm{meV}, 20 \mathrm{~K}\right]$; for Fig. 4(e), $\left[0.63 G_{0}, 1.25 G_{0}, 40 \mathrm{meV}, 3.0 \mathrm{meV}, 7.5 \mathrm{~K}\right]$. 